\title{
Graphene Oxide Liquid Crystals as a Versatile and Tunable Alignment Medium for the Measurement of Residual Dipolar Couplings in Organic Solvents
}

\author{
Xinxiang Lei, ${ }^{* \dagger, \S}$ Zhen Xu, ${ }^{\ddagger}$ Han Sun, "Shun Wang, ${ }^{\dagger}$ Christian Griesinger, ${ }^{\prime \prime}$ Li Peng, ${ }^{\ddagger}$ Chao Gao, ${ }^{*} \neq$ \\ and Ren X. Tan*,§ \\ ${ }^{\dagger}$ College of Chemistry \& Materials Engineering, Wenzhou University, Wenzhou 325035, P. R. China \\ ${ }^{\ddagger}$ MOE Key Laboratory of Macromolecular Synthesis and Functionalization, Department of Polymer Science and Engineering, \\ Zhejiang University, 38 Zheda Road, Hangzhou 310027, P. R. China \\ ${ }^{\S}$ Institute of Functional Biomolecules, State Key Laboratory of Pharmaceutical Biotechnology, Nanjing University, Nanjing 210093, P. \\ R. China \\ "Department of NMR Based Structural Biology, Max Planck Institute for Biophysical Chemistry, Am Fassberg 11, 37077 Göttingen, \\ Germany
}

Supporting Information

\begin{abstract}
Residual dipolar couplings (RDCs) have proven to be an invaluable anisotropic NMR parameter for the structural elucidation of complex biopolymers and organic molecules. However, a remaining bottleneck limiting its wider use by organic and natural product chemists is the lack of a range of easily applicable aligning media for diverse organic solvents. In this study, graphene oxide (GO) liquid crystals (LCs) were developed to induce partial orientation of organic molecules to allow RDC measurements. These LCs were determined to be maintainable at very low concentrations (as low as $1 \mathrm{mg}$ / $\mathrm{mL}$, corresponding to quadrupolar ${ }^{2} \mathrm{H}$ splittings ranging from 2.8 to $30 \mathrm{~Hz}$ and maximum ${ }^{13} \mathrm{C}-{ }^{1} \mathrm{H}$ dipolar couplings of $20 \mathrm{~Hz}$ for camphor in a $\mathrm{CH}_{3} \mathrm{COCH}_{3}$ /water system) and to be remarkably stable and broadly compatible with aqueous and organic solvents such as dimethyl sulfoxide, $\mathrm{CH}_{3} \mathrm{COCH}_{3}$, and $\mathrm{CH}_{3} \mathrm{CN}$. Moreover, compared with those for other alignment media, very clean and high-quality NMR spectra were acquired with the GO molecules in solution because of their rigidity and high molecular weight. The developed medium offers a versatile and robust method for RDC measurements that may routinize the $\mathrm{RDC}$-based structure determination of organic molecules.
\end{abstract}

Solution NMR parameters such as residual dipolar couplings $\checkmark$ (RDCs), ${ }^{1}$ NOE enhancements, ${ }^{2}$ and ${ }^{3} J$ scalar couplings ${ }^{3}$ have been widely used to determine the $3 \mathrm{D}$ structures of biopolymers and organic molecules. Among these parameters, RDCs can reflect long-range structural restraints of global character and thus have been demonstrated to improve the precision and accuracy of NMR structures of biomolecules and to provide deeper insight into their internal dynamics. ${ }^{4}$ In the past few years, RDC-enhanced structure analysis for organic molecules has attracted increasing attention because it allows the determination of the relative configuration of organic molecules in addition to constitution and conformation. ${ }^{5}$

To detect RDCs, a weak partial alignment of the analyte in the magnetic field is required. For this purpose, two main types of alignment media have been developed: ${ }^{6}$ stretched polymer gels, such as PDMS, PAN, PS, PVAc, PH, PMMA, and PEO, and liquid crystal (LC) phases, including poly $(\gamma$-benzyl Lglutamate) (PBLG), polyisocyanates, and polyacetylenes, provide the appropriate partial alignment of organic molecules. Compared with LC phases, stretched polymer gels can be scaled to generate a moderate degree of alignment; commercially available lyotropic LC phases such as PBLG are among the most widely employed media used to align small molecules. They align instantaneously. However, the main disadvantage of PBLG is that it needs to reach a critical concentration to form the LC phase, which leads to strong alignment, resulting in complications in the interpretation of the spectra. In the past few years, the Thiele group has overcome this limitation of PBLG by scaling the degree of alignment using variable-angle sample spinning $(\mathrm{VASS})^{7}$ or chemical methods. ${ }^{8}$

As of now, there are no easily applicable LC phases compatible with the widely used solvent dimethyl sulfoxide (DMSO) that would deliver RDCs in the range that the abovementioned gels would provide. Ideally, the optimal alignment medium should possess several properties: (i) sufficiently low concentration to avoid background signals and produce weak partial alignment and a degree of alignment that is scalable through simple adjustment of the concentration; (ii) high tolerance to a wide range of $\mathrm{pH}$ and temperatures and broad solvent compatibility; (iii) simple preparation of the anisotropic sample and commercial availability at low cost; and (iv) instantaneous alignment of the molecules.

Recently, graphene oxide (GO) has received widespread attention because of its fascinating properties. As a consequence

Received: June 17, 2014

Published: July 30, 2014 
of its extremely high aspect ratio (or width/thickness ratio), several groups, including ours, have confirmed its ability to spontaneously form LCs even at very dilute concentrations in both water and organic solvents. ${ }^{9}$ Aside from the use of GO LCs to align graphene sheets for advanced materials such as graphene fibers and films, we hypothesized that GO LCs would be an intriguing candidate for the development of a novel anisotropic orientation medium whose low critical concentration, interior alignment, and facile scalability comply with the criteria for an ideal orientation medium discussed above.

We prepared GO sheets by oxidizing natural graphite using $\mathrm{KMnO}_{4}$ in concentrated $\mathrm{H}_{2} \mathrm{SO}_{4}$, as described in our previous paper. ${ }^{9 b}$ The prepared GO sheets can be easily dispersed, notably in a single-layer state, in water and in polar organic solvents such as DMF and acetone. Atomic force microscopy (AFM) characterization revealed a uniform thickness of GO sheets deposited on a mica substrate [Figure S2 in the Supporting Information (SI)], confirming its single-layer dispersion state in the solvent. Typically, GO is highly asymmetrical, having an average diameter of approximately $3.5 \mu \mathrm{m}$, which leads to its tendency to form LCs when welldispersed in a liquid medium at very low concentrations. Under examination by polarized optical microscopy (POM), GO dispersions exhibited Schlieren optical textures at concentrations as low as $1 \mathrm{mg} / \mathrm{mL}$, indicating the intrinsic orientational order. As expected, this orientational order should make GO an ideal medium to weakly align small organic molecules for RDC measurements.

As a first step, we studied the GO LCs using NMR spectroscopy in the aqueous state (Figure 1). The threshold for

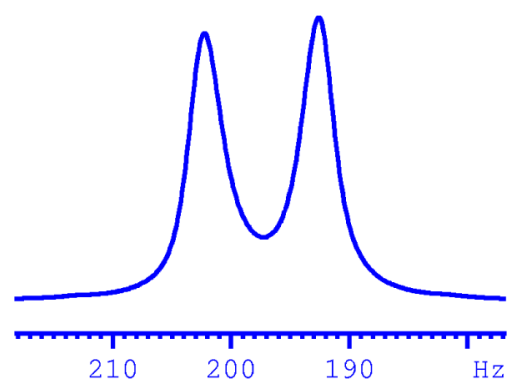

Figure 1. ${ }^{2} \mathrm{H}$ NMR spectrum $\left(76.8 \mathrm{MHz},{ }^{1} \mathrm{H}\right.$ NMR frequency 500 $\mathrm{MHz})$ of the anisotropic sample $(2 \mathrm{mg} / \mathrm{mL}$ GO LC phase $)$ in $\mathrm{D}_{2} \mathrm{O}$ and trace DMSO. A ${ }^{2} \mathrm{H}$ quadrupolar splitting of $9.61 \mathrm{~Hz}$ for DMSO was obtained. ${ }^{10}$

LC formation was detected by observing the quadrupolar coupling of the ${ }^{2} \mathrm{H}$ NMR signal of the deuterated solvent accompanied by the disappearance of the isotropic signal. It enters the LC phase at a critical GO concentration of $1 \mathrm{mg} / \mathrm{mL}$ in water. The quadrupolar splitting of the deuterated solvent signal was $2.83 \mathrm{~Hz}$, which was also confirmed by the direct observation of strong birefringence with crossed polarizers using the naked eye (Figure 2B). Similar to other LC phases, the preparation of the anisotropic sample using GO LCs is fast and straightforward, requiring only addition of the GO LC stock solution into the NMR tubes with the test samples and shaking and centrifugation in alternating fashion to achieve homogeneity (Figure 2A).

To demonstrate the alignment properties of the GO LCs, sucrose was selected because it has been well-established as a test molecule for RDC measurements in previous studies. ${ }^{61}$ Sucrose $(10 \mathrm{mg})$ was added to the GO LCs $(2 \mathrm{mg} / \mathrm{mL}$ in

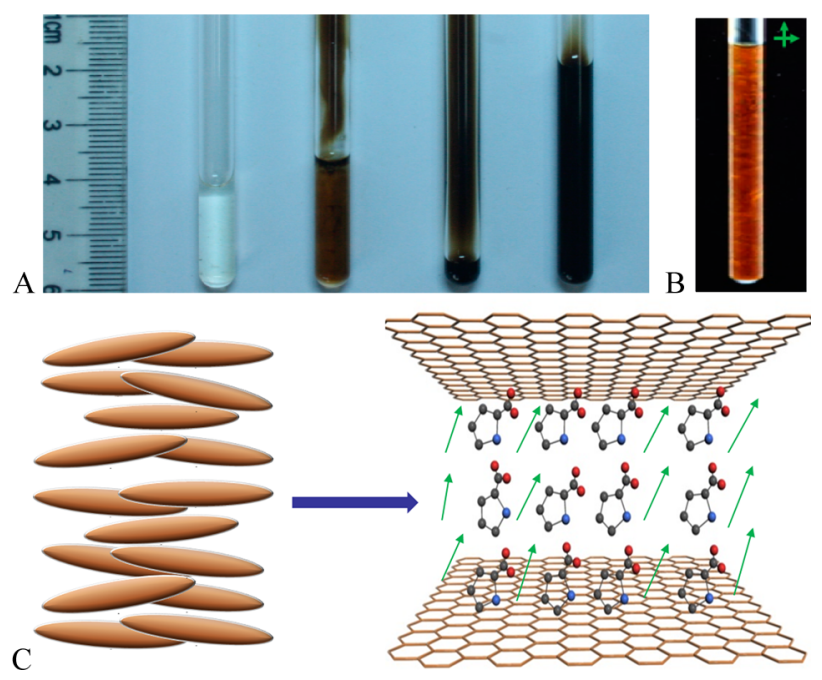

Figure 2. (A) Preparation of the alignment medium of the test sample for RDC measurements. From left to right: sample with solvent, addition of the GO LC stock solution, mixing of the GO LCs and solvent by shaking, and the GO LC sample for RDC acquisition after centrifugation. (B) Macroscopic photographs of GO aqueous dispersions in test NMR tubes between crossed polarizers. (C) Proposed model of partial orientation of the solute induced by the GO LCs.

water) with a quadrupolar $\mathrm{D}_{2} \mathrm{O}$ splitting of $6.81 \mathrm{~Hz}$. Because of the homogeneity of the GO LC sample, a high-quality ${ }^{1} \mathrm{H}$ NMR spectrum of sucrose was acquired under anisotropic conditions (Figure 3). Furthermore, in contrast to other

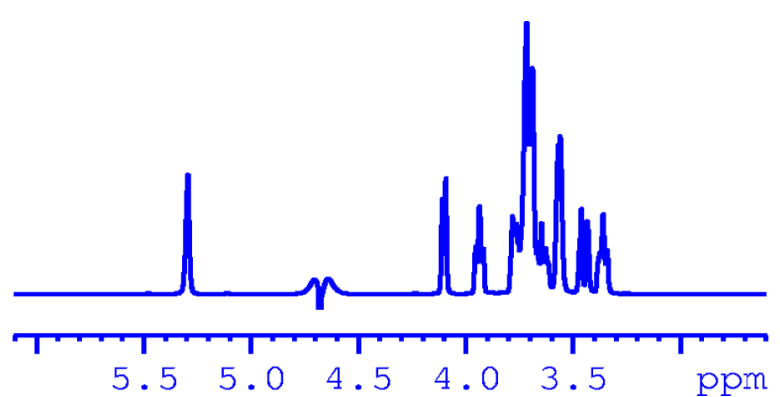

Figure 3. A portion of the $500 \mathrm{MHz}{ }^{1} \mathrm{H}$ spectrum of D-sucrose in 2 $\mathrm{mg} / \mathrm{mL}$ GO LCs using a NOESYPR1D pulse sequence for water suppression.

existing alignment media, no background signals originating from GO were observed because the critical concentration of GO to form LC phases is very low and the GO molecule itself is rigid and has a very high molecular weight. Therefore, NMR signals originating from GO are beyond the solution NMR detection limit. To acquire RDCs of sucrose, CLIP-HSQC spectra ${ }^{11}$ were recorded in an isotropic solution as well as in the GO LC phase. ${ }^{1} D_{\mathrm{C}, \mathrm{H}}$ RDCs were extracted as the difference of the couplings measured under isotropic and anisotropic conditions. The sizes of the measured RDCs of sucrose ranged from -5 to $18 \mathrm{~Hz}$, allowing their accurate measurement while avoiding artifacts from the strong induction of anisotropy.

Moreover, to explore the scope of GO LCs in aqueous solutions for the acquisition of RDCs, we also used proline as a test molecule. CLIP-HSQC spectra were recorded in an isotropic solution and in GO solution for the RDC measurements (Figure 4). The alignment tensor of proline was 


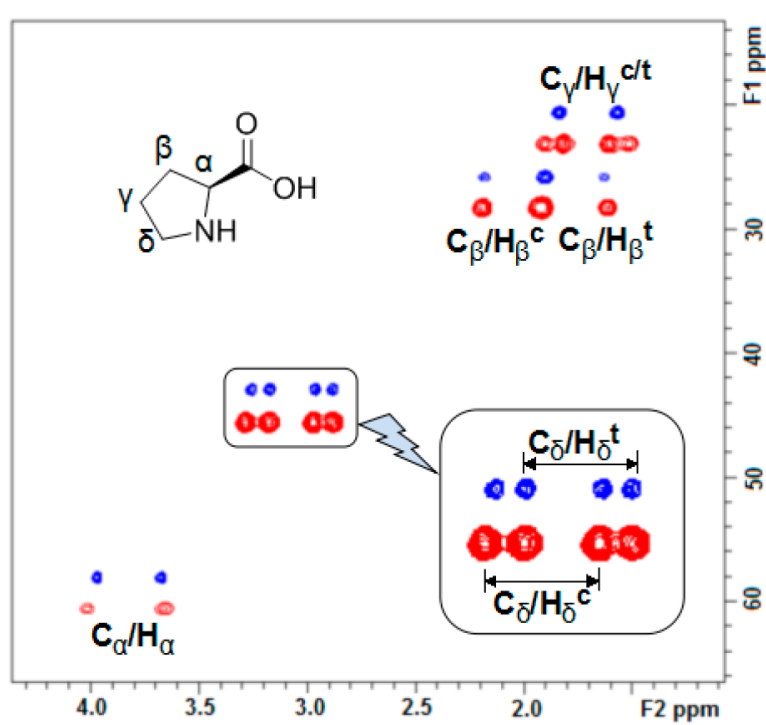

Figure 4. A portion of the $500 \mathrm{MHz}{ }^{1} \mathrm{H},{ }^{13} \mathrm{C}$-CLIP-HSQC spectra of Lproline in the isotropic $\mathrm{D}_{2} \mathrm{O}$ phase (blue) and in anisotropic $3 \mathrm{mg} / \mathrm{mL}$ GO LC solution (red).

calculated from the measured RDCs using the singular value decomposition (SVD) method ${ }^{12}$ with the MSpin program package (http://www.mestrelab.com). ${ }^{13}$ The structure for the RDC fitting was minimized using density functional theory (DFT) calculations (see the SI). The linear relation between the experimental and back-calculated RDC values is shown in Table S2 in the SI. An excellent quality factor ( $Q$ factor) of 0.046 for the regression coefficient was obtained, indicating that the measurement of the RDC data using the GO LC phases was precise.

To address the question of compatibility of GO LCs with a wide range of solvents, we extensively surveyed whether the aqueous GO LCs could function as an alignment medium to acquire RDCs in organic solvents. First, by monitoring the ${ }^{2} \mathrm{H}$ NMR spectra, we observed deuterium quadrupolar splittings of 27.00, 30.71, and $23.89 \mathrm{~Hz}$ when GO LCs were mixed at a critical concentration of $3.8 \mathrm{mg} / \mathrm{mL}$ with organic solvents DMSO, acetone, and $\mathrm{CH}_{3} \mathrm{CN}$, respectively (Figure S4). The ability of the aqueous GO LC phases to mix with polar organic solvents allows the RDC measurements to be performed using intermediate polar organic compounds. Notably, the quadrupolar splittings observed in the ${ }^{2} \mathrm{H}$ NMR spectrum scale up with the concentration of GO (Figure 5), indicating that the strength of the alignment induced by GO LCs can be scaled through adjustment of its concentration. The ${ }^{2} \mathrm{H}$ quadrupolar splitting becomes unresolved at a GO concentration of $0.5 \mathrm{mg} /$ $\mathrm{mL}$, and poor resolution is seen beyond the concentration of 4 $\mathrm{mg} / \mathrm{mL}$ in $\mathrm{DMSO} /$ water systems because of the viscosity (see the SI). The line broadening of the solute molecules also becomes severe with increasing GO concentration (Figure 5), which is a main drawback of the introduced medium.

According to the literature, ${ }^{9 \mathrm{~d}} \mathrm{GO}$ sheets can be dispersed in pure organic solvents below a certain concentration and exhibit an LC phase. As a result of the dispersibility of GO in organic solvents such as DMSO, acetone, and acetonitrile (its proportion can reach $80 \%$ ), the viscosity of the GO LCs phase can become so high that the quadrupolar ${ }^{2} \mathrm{H}$ splitting cannot be resolved because of poor shimming of the spectrometer.

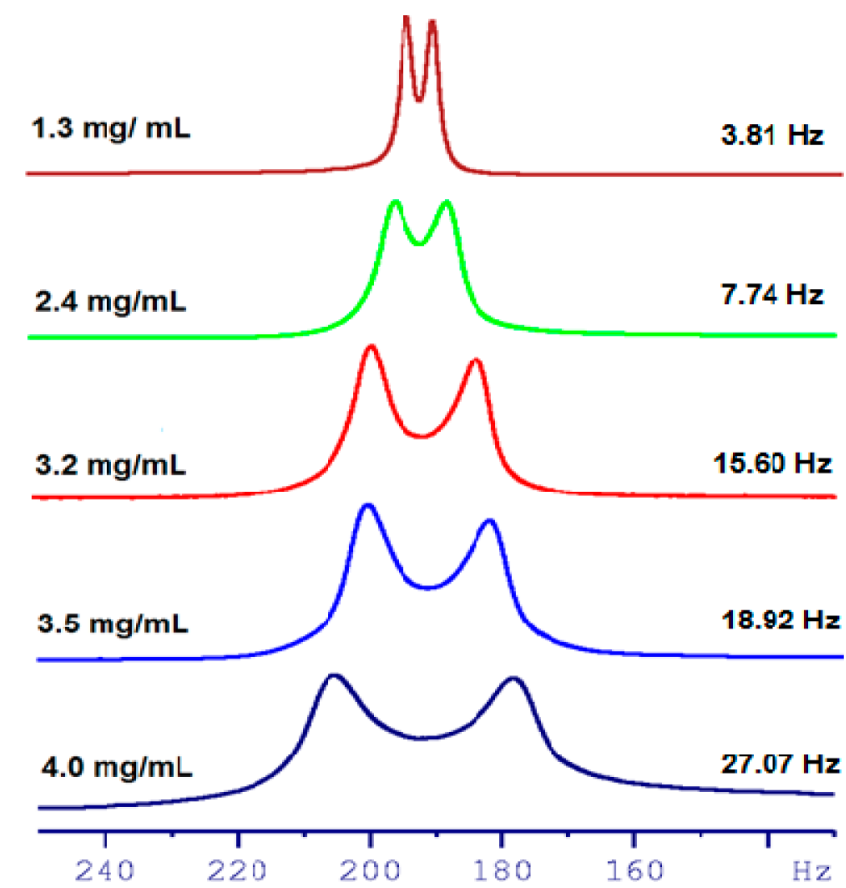

Figure 5. $1 \mathrm{D}{ }^{2} \mathrm{H}$ spectra $\left(25{ }^{\circ} \mathrm{C}\right.$, eight scans $)$ of samples containing (top to bottom) 1.3, 2.4, 3.2, 3.5, and $4 \mathrm{mg} / \mathrm{mL} \mathrm{GO}$ in $1: 4: 5 \mathrm{D}_{2} \mathrm{O}$ / $\mathrm{H}_{2} \mathrm{O} /$ DMSO.

These encouraging results prompted us to immediately test the feasibility of GO LCs for RDC measurements of organic molecules. Camphor and menthol were selected as analytes to assess the alignment properties of the GO LCs in various organic solvent mixtures with $50 \%$ water. In a $2 \mathrm{mg} / \mathrm{mL}$ GO $\mathrm{LC}$ anisotropic medium, the range of the RDCs for camphor and menthol was -20 to $25 \mathrm{~Hz}$, which was extracted easily and precisely. Remarkably, the plots of experimental versus backcalculated RDCs of the camphor structure in DMSO, acetone, and $\mathrm{CH}_{3} \mathrm{CN}$ show excellent correlations, with correlation factors $(R)$ of $0.983,0.990$, and 0.998 , respectively, and $Q$ factors of $0.147,0.142$, and 0.055 , respectively (Figure 6). The measured and calculated RDCs of menthol are also in good agreement (Figure S9), with $R=0.979,0.968$, and 0.991 for menthol samples dispersed in DMSO, acetone, and $\mathrm{CH}_{3} \mathrm{CN}$, respectively.

In addition to their high compatibility with polar organic solvents, the GO LCs exhibit excellent tolerance to $\mathrm{pH}$, high ionic strength, and a wide range of temperatures. The ${ }^{2} \mathrm{H}$ splitting is well-resolved in environments ranging from $\mathrm{pH} 1$ to 14 and in a $200 \mathrm{mM} \mathrm{NaCl}$ solution; the ${ }^{2} \mathrm{H}$ splitting is also stable at temperatures ranging from 5 to $80{ }^{\circ} \mathrm{C}$ (see the SI). Additionally, the GO LCs are inert and stable even after several months of storage under ambient conditions.

In summary, we have shown that GO LCs are a versatile and effective medium to induce moderate degrees of alignment for the purpose of conducting RDC measurements on organic molecules. This work represents the first example of the use of carbon-based LCs for measuring anisotropic NMR parameters. GO LCs have several unrivaled advantages in comparison with other media previously used. First, they produce clean NMR spectra without background signals, which enables measurements on rare natural products, where the limited availability of the sample constitutes a methodological challenge for the RDC measurements. In previous studies, such samples were 


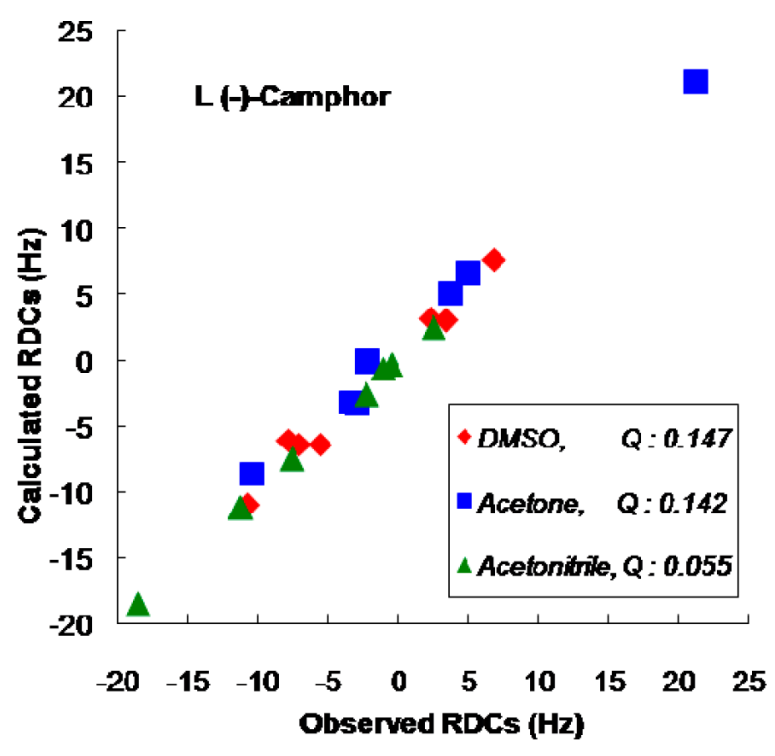

Figure 6. Correlation between the observed ${ }^{1} D_{\mathrm{C}, \mathrm{H}} \mathrm{RDCs}$ and the RDCs calculated for the DFT structure of $\mathrm{L}-(-)$-camphor in GO LC phases with $50 \% \mathrm{D}_{2} \mathrm{O}$ and $50 \%$ DMSO, acetone, or acetonitrile. The RDC fitting was performed by SVD. The $Q$ factors are $0.147,0.142$, and 0.055 , respectively.

measured by deuteration of the alignment media ${ }^{14}$ or the use of a relaxation filter to suppress the background signals. ${ }^{6 c}$ The GO LCs are compatible with the use of $1.7 \mathrm{~mm}$ cryoprobes for optimal sensitivity. Second, because GO LCs are an adjustable alignment medium, they also allow for the precise and accurate acquisition of RDCs through simple variation of the concentration. Third, GO LCs are extraordinarily stable, insensitive to $\mathrm{pH}$, salt, and temperature, and compatible with different polar solvents, making them complementary to PBLG, which is mainly compatible with apolar solvents. Fourth, the preparation of the anisotropic alignment sample is straightforward, requiring only mixing of the solute with the stock GO LCs solution, which can be stored for several months under ambient conditions. Finally, GO LCs can be readily produced on a large scale and can potentially be made commercially available at low cost. We believe that the presented GO LCs will significantly facilitate the collection of RDCs and provide a new path to an everyday laboratory approach for the routine measurement of RDCs.

\section{ASSOCIATED CONTENT}

\section{S Supporting Information}

Experimental details; data for sucrose, proline, camphor, and menthol; and a description of GO LC preparation. This material is available free of charge via the Internet at http:// pubs.acs.org.

\section{AUTHOR INFORMATION}

\section{Corresponding Authors}

xxlei@wzu.edu.cn

chaogao@zju.edu.cn

rxtan@nju.edu.cn

\section{Notes}

The authors declare no competing financial interest.

\section{ACKNOWLEDGMENTS}

This work was financed by Zhejiang Provincial Natural Science Foundation of China under Grant LY14B020010, LY12B02007 and by NSFC under Grant 21325417. C.G. was supported by the Max Planck Society and the DFG (FOR 934). We thank Prof. Burkhard Luy for providing the CLIP-HSQC pulse program.

\section{REFERENCES}

(1) (a) Tolman, J. R.; Flanagan, J. M.; Kennedy, M. A.; Prestegard, J. H. Proc. Natl. Acad. Sci. U.S.A. 1995, 92, 9279. (b) Tolman, J. R.; Flanagan, J. M.; Kennedy, M. A.; Tjandra, N.; Bax, A. Science 1997, 278, 1111. (c) de Alba, E.; Tjandra, N. Prog. NMR Spectrosc. 2002, 40, 175 .

(2) Anet, F. A. L.; Bourn, A. J. R. J. Am. Chem. Soc. 1965, 87, 5250.

(3) Karplus, M. J. Chem. Phys. 1959, 30, 11.

(4) (a) Blackledge, M. Prog. Nucl. Magn. Reson. Spectrosc. 2005, 46, 23. (b) Lange, O. F.; Lakomek, N. A.; Farès, C.; Schröder, G. F.; Walter, K. F.; Becker, S.; Meiler, J.; Grubmüller, H.; Griesinger, C.; de Groot, B. L. Science 2008, 320, 1471.

(5) Gil, R. R. Angew. Chem., Int. Ed. 2011, 50, 7222.

(6) (a) Freudenberger, J. C.; Spiteller, P.; Bauer, R.; Kessler, H.; Luy, B. J. Am. Chem. Soc. 2004, 126, 14690. (b) Haberz, P.; Farjon, J.; Griesinger, C. Angew. Chem., Int. Ed. 2005, 44, 427. (c) Kummerlöwe, G.; Auernheimer, J.; Lendlein, A.; Luy, B. J. Am. Chem. Soc. 2007, 129, 6080. (d) Thiele, C. M. Concepts Magn. Reson. 2007, 30A, 65. (e) Thiele, C. M. Eur. J. Org. Chem. 2008, 5673. (f) Kummerlöwe, G.; Luy, B. TrAC, Trends Anal. Chem. 2009, 28, 483. (g) Arnold, L.; Marx, A.; Thiele, C. M.; Reggelin, M. Chem.-Eur. J. 2010, 16, 10342. (h) Gayathri, C.; Tsarevsky, N. V.; Gil, R. R. Chem.-Eur. J. 2010, 16, 3622. (i) Thiele, C. M.; Pomerantz, W. C.; Abbott, N. L.; Gellman, S. H. Chem. Commun. 2011, 47, 502. (j) Dama, M.; Berger, S. Org. Lett. 2012, 14, 241. (k) Meyer, N. C.; Krupp, A.; Schmidts, V.; Thiele, C. M.; Reggelin, M. Angew. Chem., Int. Ed. 2012, 51, 8334. (1) Montag, T.; Thiele, C. M. Chem.-Eur. J. 2013, 19, 2271. (m) Merle, C.; Kummerlöwe, G.; Freudenberger, J. C.; Halbach, F.; Stöwer, W.; Gostomski, C. L.; Höpfner, J.; Beskers, T.; Wilhelm, M.; Luy, B. Angew. Chem., Int. Ed. 2013, 52, 10309.

(7) Thiele, C. M. Angew. Chem., Int. Ed. 2005, 44, 2787.

(8) (a) Marx, A.; Thiele, C. Chem.-Eur. J. 2009, 15, 254. (b) Marx, A.; Böttcher, B.; Thiele, C. M. Chem.-Eur. J. 2010, 16, 1656.

(9) (a) Kim, J. E.; Han, T. H.; Lee, S. H.; Kim, J. Y.; Ahn, C. W.; Yun, J. M.; Kim, S. O. Angew. Chem., Int. Ed. 2011, 50, 3043. (b) Xu, Z.; Gao, C. ACS Nano 2011, 5, 2908. (c) Xu, Z.; Gao, C. Nat. Commun. 2011, 2, 571. (d) Jalili, R.; Aboutalebi, S. H.; Esrafilzadeh, D.; Konstantinov, K.; Moulton, S. E.; Razal, J. M.; Wallace, G. G. ACS Nano 2013, 7, 3981.

(10) In aqueous solution, the ${ }^{2} \mathrm{H}$ splitting of $\mathrm{D}_{2} \mathrm{O}$ disappeared when the $\mathrm{pH}$ was tuned below 7, but trace deuterated organic solvents (e.g., DMSO) were added and showed good splitting.

(11) Enthart, A.; Freudenberger, J. C.; Furrer, J.; Kessler, H.; Luy, B. J. Magn. Reson. 2008, 192, 314.

(12) Losonczi, J. A.; Andrec, M.; Fischer, M. W. F.; Prestegard, J. H. J. Magn. Reson. 1999, 138, 334.

(13) Navarro-Vazquez, A. Magn. Reson. Chem. 2012, 50, S73.

(14) Kummerlöwe, G.; Knör, S.; Frank, A. O.; Paululat, T.; Kessler, H.; Luy, B. Chem. Commun. 2008, 5722. 